\title{
Effect of double-pass and single-pass architecture in Brillouin-Raman fiber laser
}

\begin{abstract}
We experimentally investigate effect of double pass and single pass configuration in multiwavelength Brillouin-Raman fiber laser in a linear cavity. Up to 110 flat amplitude multiwavelengths Brillouin Stokes with spacing of $0.16 \mathrm{~nm}$ within wavelength range of 1554 to $1570 \mathrm{~nm}$ in single pass configuration are generated. The generated wavelengths with OSNR $25 \mathrm{~dB}$ compared with that of double pass architecture. Single space configuration has a superior performance due to double line spacing, higher OSNR, wider bandwidth and high stability than that of double pass configuration. Lasing lines in double pass has $0.08 \mathrm{~nm}$ space due to the increment of Rayleigh scattering in double pass configuration.
\end{abstract}

Keyword: Brillouin spectra; Raman lasers; Fibre lasers; Laser cavity resonators; Spectral line breadth 